\title{
Determining Two-Sided Surface Profiles of Micro-Optical Elements Using a Dual-Wavelength Digital Holographic Microscope With Liquids
}

\author{
Hong Seok Lee', Sanghoon $\mathrm{Shin}^{2}$, Heonjoo Lee ${ }^{3}$, and Younghun $\mathrm{Yu}^{3 *}$ \\ ${ }^{I}$ Department of Physics, Chonbuk National University, Jeonju 561-756 Korea \\ ${ }^{2}$ KPS, Hwaseong 445-811, Korea \\ ${ }^{3}$ Department of Physics, Jeju National University, Jeju 690-756, Korea
}

(Received June 17, 2014 : revised September 25, 2014 : accepted September 25, 2014)

\begin{abstract}
In this paper, a method is proposed for simultaneously measuring the front and back surface profiles of transparent micro-optical components. The proposed method combines a dual-wavelength digital holographic microscope with liquids to record holograms at different wavelengths, and then numerically reconstructs the three-dimensional phase information to image the front and back sides of the sample. A theoretical model is proposed to determine the surface information, and imaging of an achromatic lens is demonstrated experimentally. Unlike conventional interferometry, our proposed method supports nondestructive measurement and direct observation of both front and back profiles of micro-optical elements.
\end{abstract}

Keywords : Diffractive optics, Holography, Image reconstruction

OCIS codes : (090.0090) Holography; (100.3010) Image reconstruction techniques; (050.1970) Diffractive optics

\section{INTRODUCTION}

Holography is a technique for recording phase modulation using interference patterns formed when light is reflected or transmitted by an object onto a photoplate. The recording requires object and reference beams, the combination of which generates the interference pattern. In the past, holographic interference patterns were recorded on film plates; presently, however, charge-coupled devices (CCDs) or complementary metal oxide semiconductor (CMOS) cameras are commonly used, and a computer reconstructs the hologram. Yaroslavskii et al. proposed a method for numerical hologram reconstruction in the 1970s, and Ounral and Scott used numerical reconstruction to measure the size of a particle, after improving on the reconstruction algorithm [1-6]. This method of digitally recording and reconstructing a numerical hologram is known as digital holography [7-10].

There are two types of digital holographic microscopes (DHMs): reflection and transmission. Typical optical components have two surfaces, front and back. However, transmission interferometers or DHMs cannot measure both three-dimensional (3D) surface profiles simultaneously, because the output from the optical components includes integrated surface information.
In reflection interferometry, the phase information of each surface is measured separately and then numerically reconstructed to provide 3D information about the sample. Thus reflection interferometry is used to measure both $3 \mathrm{D}$ surface profiles. However, these approaches rely on movable mechanisms and accurate position determination to obtain all of the phase information. Low-coherence interferometry (LCI) could measure the surfaces by controlling the reference optical length as in optical coherence tomography, but LCI requires many images to obtain all of the phase information [11, 12].

In this paper we propose a method for simultaneously measuring the front and back surface profiles of transparent micro-optical components, using a dual-wavelength DHM [13-17] and liquids. We also propose a theoretical model for determining the surface information, and experimentally demonstrate the use of this model for an achromatic lens.

\section{THEORETICAL MODEL}

\subsection{Hologram Recording and Reconstruction}

In the hologram recording process, a plane reference wave $R$ and an object wave $O$ interfere at the CCD. The hologram

\footnotetext{
*Corresponding author: yyhyoung@jejunu.ac.kr

Color versions of one or more of the figures in this paper are available online.
} 
intensity is given by

$$
I_{H}(x, y)=|R|^{2}+|O|^{2}+R^{*} O+R O^{*},
$$

where $R^{*}$ and $O^{*}$ denote the complex conjugates of the reference and object waves respectively $[1,2]$. A digital holographic image can be recorded using a monochromatic CCD camera. The digital hologram $I_{H}(k, l)$ is an $N \times N$ array resulting from two-dimensional sampling of $I_{H}(x, y)$ by the CCD camera, expressed as follows:

$$
I_{H}(k, l)=I_{H}(x, y) \operatorname{rect}\left(\frac{x}{L}, \frac{y}{L}\right) \times \sum_{k=-N / 2}^{N / 2} \sum_{l=-N / 2}^{N / 2} \delta(x-k \Delta x, y-l \Delta y),
$$

where $k$ and $l$ are integers, $L \times L$ denotes the area of the CCD chip, and $\triangle x$ and $\triangle y$ indicate the horizontal and vertical pixel sizes of the CCD.

If a screen is placed at a distance $d$ behind the hologram, a real image is formed on the screen. Mathematically the amplitude and phase distributions in the plane of the real image can be calculated using the Fresnel-Kirchhoff integral $[1,2]$. If a plane wave illuminates the hologram with an amplitude transmittance $I_{H}(x, y)$, the Fresnel-Kirchhoff integral yields a complex amplitude $\Psi(\xi, \eta)$ in the real image plane as

$$
\begin{aligned}
\Psi(\xi, \eta) & =\frac{\exp (i 2 \pi d / \lambda)}{i \lambda d} \exp \left[\frac{i \pi}{d \lambda}\left(\xi^{2}+\eta^{2}\right)\right] \\
& \times \iint I_{H}(x, y) \exp \left[\frac{i \pi}{d \lambda}\left(x^{2}+y^{2}\right)\right] \\
& \times \exp \left[\frac{i 2 \pi}{d \lambda}(\xi x+\eta y)\right] d x d y
\end{aligned}
$$

where $\lambda$ is the wavelength and $d$ is the reconstruction distance. From Eq. (3), the Fresnel-Kirchhoff integral can be considered as the Fourier transformation of the function $I_{H}(x, y) \exp \left[i \pi\left(x^{2}+y^{2}\right) / \lambda d\right]$ at the spatial frequencies $\xi$ and $\eta$. Because $\Psi(\xi, \eta)$ is an array of complex numbers, one can obtain an amplitude-contrast image using the intensity:

$$
I(\xi, \eta)=\operatorname{Re}[\Psi(\xi, \eta)]^{2}+\operatorname{Im}[\Psi(\xi, \eta)]^{2}
$$

The quantitative phase image is obtained by calculating the argument [18-21]

$$
\Psi(\xi, \eta)=\arctan \left\{\frac{\operatorname{Im}[\Psi(\xi, \eta)]}{\operatorname{Re}[\Psi(\xi, \eta)]}\right\} .
$$

The real 3D information is acquired by phase-unwrapping the quantitative phase image.

\subsection{Optical Path Difference in A Dual-Wavelength Digital Holographic Microscope with Liquids}

Consider the optical-path difference of a beam traveling through a transparent sample of refractive index $n_{L}$ that is immersed in liquids with refractive indices $n_{1}$ and $n_{2}$ (see Fig. 1). The optical path length between planes $A$ and $B$ of the test surface can be described as [22]:

$$
\begin{aligned}
& O P L_{0}=Z_{01} n_{1}+n_{L} t+Z_{02} n_{2} \\
& O P L(x, y)=Z_{1}(x, y) n_{1}+L_{1}(x, y) n_{L}+n_{L} t+Z_{2}(x, y) n_{2}+L_{2}(x, y) n_{L} \\
& O P D(x, y)=\left[Z_{1}(x, y)-Z_{01}\right] n_{1}+\left[Z_{2}(x, y)-Z_{02}\right] n_{2}+\left[L_{1}(x, y)+L_{2}(x, y)\right] n_{L},
\end{aligned}
$$

where $Z_{0 i}$ and $Z_{i}(x, y)$ represent the vertex and surface height, respectively, and $L_{i}(x, y)$ is the distance traveled from the surface to the vertex $(i=1,2) . \operatorname{OPD}(x, y)$ is the path difference between $O P L$ and $O P L_{0}$. The planes $A$ and $B$ are parallel, so the distance between them is constant. This determines the relationship between $Z$ and $L$ :

$$
\begin{aligned}
& L_{1}=Z_{01}-Z_{1}(x, y) \\
& L_{2}=Z_{02}-Z_{2}(x, y),
\end{aligned}
$$

$$
\text { Substituting Eq. (7) into Eq. (6) gives }
$$

$$
O P D(x, y)=-L_{1}(x, y) n_{1}-L_{2}(x, y) n_{2}+\left[L_{1}(x, y)+L_{2}(x, y)\right] n_{L} .
$$

Equation (8) includes the integrated surface information $\left[L_{1}(x, y)+L_{2}(x, y)\right]$ of the sample, not information about the individual surface profiles. Equation (8), therefore, represents the coupled information of the surface profiles and can be modified to include the effect of different wavelengths as follows:

$$
\begin{aligned}
& O P D_{1}(x, y)=-L_{1}(x, y) n_{11} \lambda_{1}-L_{2}(x, y) n_{21} \lambda_{1}+\left[L_{1}(x, y)+L_{2}(x, y)\right] n_{L 1} \lambda_{1} \\
& O P D_{2}(x, y)=-L_{1}(x, y) n_{12} \lambda_{2}-L_{2}(x, y) n_{22} \lambda_{2}+\left[L_{1}(x, y)+L_{2}(x, y)\right] n_{L 2} \lambda_{2},
\end{aligned}
$$

$O P D_{i}(x, y)(i=1,2)$ is the optical path difference at wavelength $\lambda_{i}$, while $n_{l i}, n_{2 i}$ and $n_{L i}$ represent the refractive index of liquid and sample at wavelength $\lambda_{i}$, respectively. From Eq. (9) we find that the sets of information for individual surfaces, $L_{I}(x, y)$ and $L_{2}(x, y)$, are decoupled. The surface profile information in $L_{l}(x, y)$ and $L_{2}(x, y)$ can also be expressed as in Eq. (10):

$$
\begin{aligned}
& L_{1}(x, y)+L_{2}(x, y)=\frac{O P D_{1}(x, y)+L_{1}(x, y) n_{11} \lambda_{1}+L_{2}(x, y) n_{21} \lambda_{1}}{n_{L 1} \lambda_{1}} \\
& L_{1}(x, y)+L_{2}(x, y)=\frac{O P D_{2}(x, y)+L_{1}(x, y) n_{12} \lambda_{2}+L_{2}(x, y) n_{22} \lambda_{2}}{n_{L 2} \lambda_{2}}
\end{aligned}
$$

The result above is obtained from the expression for the optical-path difference with two wavelengths and two different liquids. Equation (10) presents the decoupled surface profile 


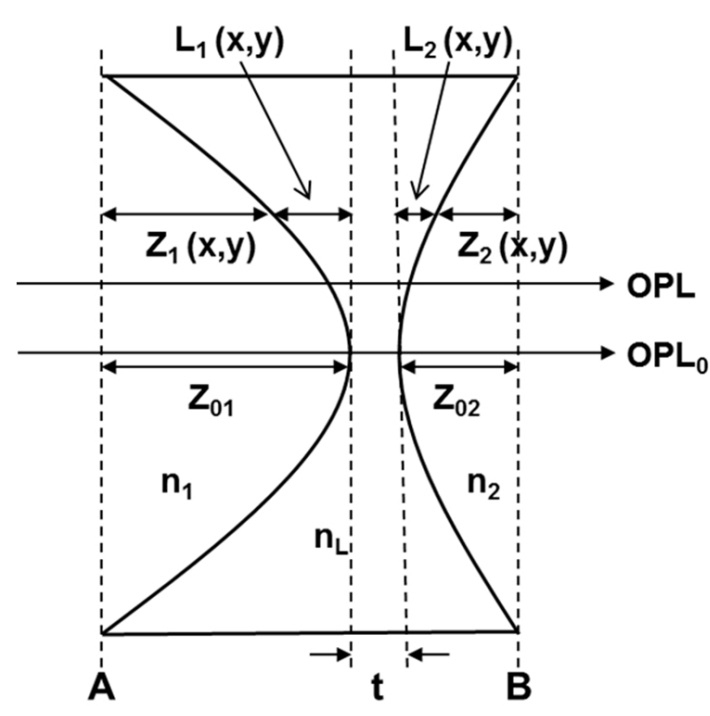

FIG. 1. Schematic diagram of a doubly concave lens immersed in liquids.

information, and shows that both surface profiles of a transmission optical component can be measured simultaneously using a dual-wavelength DHM and two different liquids.

\section{EXPERIMENT AND RESULTS}

Figure 2 shows a schematic diagram of the experimental setup for a dual-wavelength transmission holographic microscope with liquids, for measuring both surfaces simultaneously. The basic experimental setup is similar to that of a MachZehnder type interferometer. A $10-\mathrm{mW}$ He-Ne laser (633 $\mathrm{nm})$ and laser diode $(532 \mathrm{~nm})$ were used as the light sources. Water and a refractive index liquid (Cargille Laboratories) were used. The refractive index of the liquid was 1.6171 and 1.6317 at 633 and $532 \mathrm{~nm}$, respectively, while that of water was 1.33168 and 1.33372 at 633 and $532 \mathrm{~nm}$, respectively. Different liquids are filled as in Fig. 3.

We used a CCD camera (Imperx) to record the holograms. The pixel size and the number of pixels were $7.4 \times 7.4 \mu \mathrm{m}$ and $1024 \times 1024$, respectively. Achromatic lenses (Edmund Optics) were used as the samples, each with a different radius of curvature (ROC) 26.82 and $61.38 \mathrm{~mm}$; and refractive indices of 1.51508 and 1.51947 at 633 and $532 \mathrm{~nm}$, respectively.

Figure 4 shows the experimental results for the achromatic lens from dual-wavelength DHM with liquids. Figures 4(a) and (b) show the holograms at 532 and $633 \mathrm{~nm}$ respectively. Figures 4(c) and (d) show respectively the reconstructed quantitative phase image of each hologram with 3D gray-level surface profiles of the samples shown in Figs. 4(e) and (f) for 532 and $633 \mathrm{~nm}$. To acquire the 3D gray-level images, we used Flynn's unwrapping method [23].

Figure 5(a) illustrates the gray levels measured along the dashed lines in Fig. 4(e) and Fig. 4(f). The squares and circles correspond to the results for wavelengths of 532 and 633

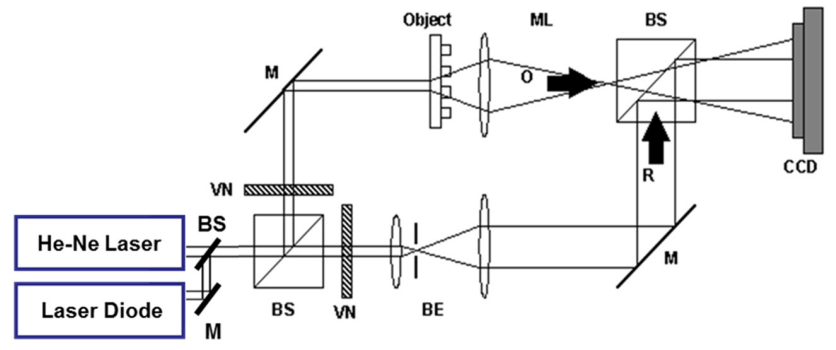

FIG. 2. Transmission-type dual-wavelength digital holographic microscope (DHM) (VN: variable-density filter; BS: beam splitter; M: mirror; O: object wave; R: reference wave; BE: beam expander with spatial filter; ML: microscope objective lens; CCD: charge-coupled device).

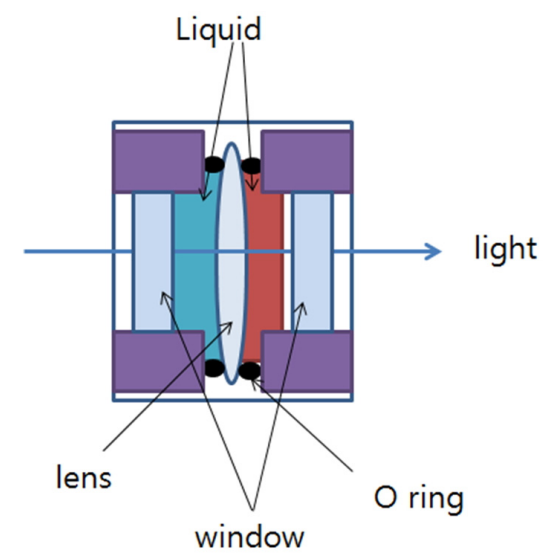

FIG. 3. Lens holder for filling liquids before and after the lens.

$\mathrm{nm}$, respectively. The difference between the profiles for each wavelength was very small, as predicted by Eq. (9). The OPD includes information about the lens shape and refractive index; however, the OPD in Fig. 5(a) is not the actual height. The OPD difference between 532 and 633 $\mathrm{nm}$ is caused by the difference in the refractive indices. We calculated the front and back profiles using Eq. (10) and data from Figs. 4(e) and (f). The calculated results for the 3D color image from each surface are shown in Figs. 5 (b) and (c). Note that the optical-path difference between the wavelengths, as well as noise, affected the calculated image. In Fig. 5(d) we show the spatial profiles indicated by the dashed lines in Figs. 5(b) and (c); the squares and circles correspond to the raw data, while the lines represent the fitted data. The fitted data show that the profile of each surface is different, and the surface information is decoupled. Next, we calculated the ROC from the calculated profiles. The ROC can be found from the completely reconstructed surface by fitting to a best-fit sphere using singular-value decomposition (SVD). Any spherical surface along the $z$-axis can be expressed as

$$
a\left(x^{2}+y^{2}+z^{2}\right)+b z=1,
$$




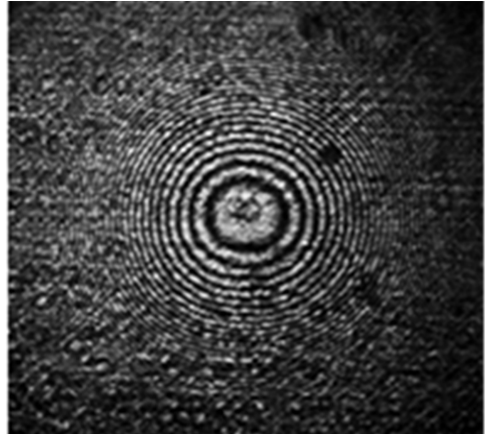

(a)

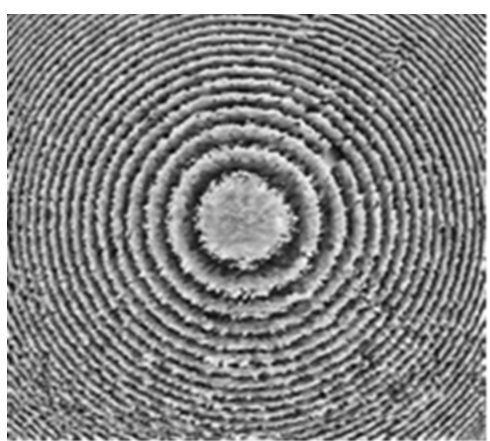

(d)

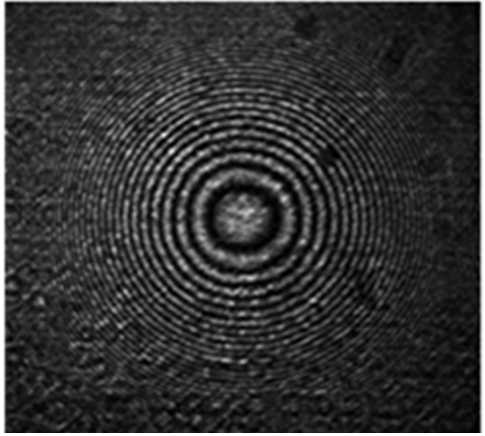

(b)

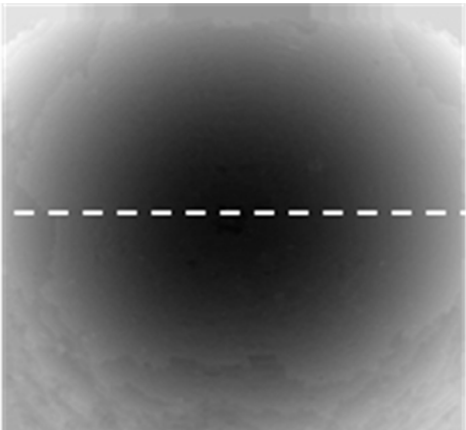

(e)

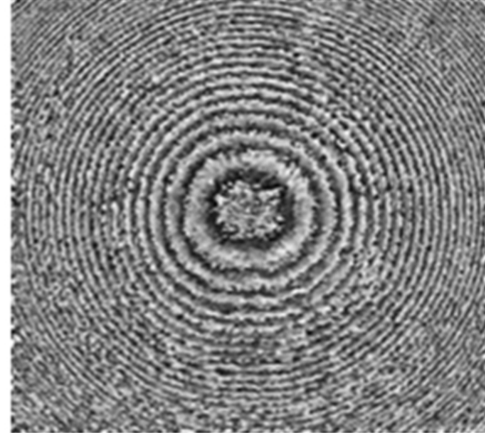

(c)

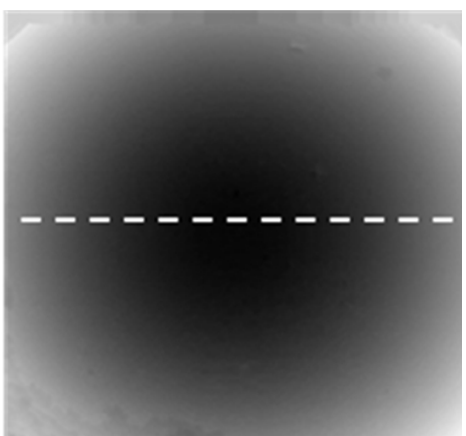

(f)

FIG. 4. Experimental results from dual-wavelength DHM with liquids. Holograms for wavelengths of (a) $532 \mathrm{~nm}$ and (b) $633 \mathrm{~nm}$. Reconstructed quantitative phase image for (c) $532 \mathrm{~nm}$ and (d) $633 \mathrm{~nm}$. 3D gray-level images for (e) $532 \mathrm{~nm}$ and (f) $633 \mathrm{~nm}$.

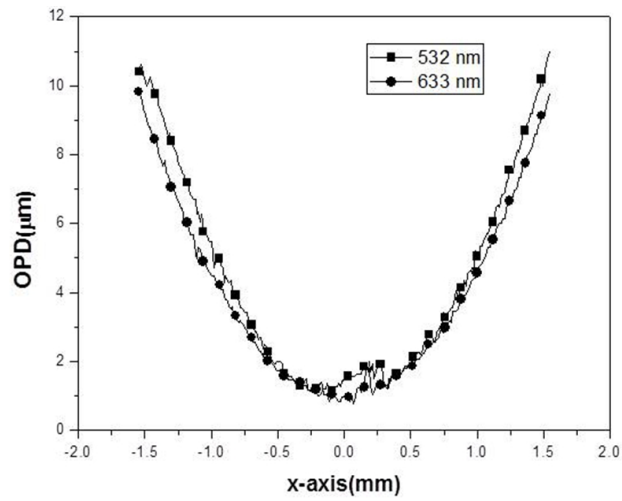

(a)

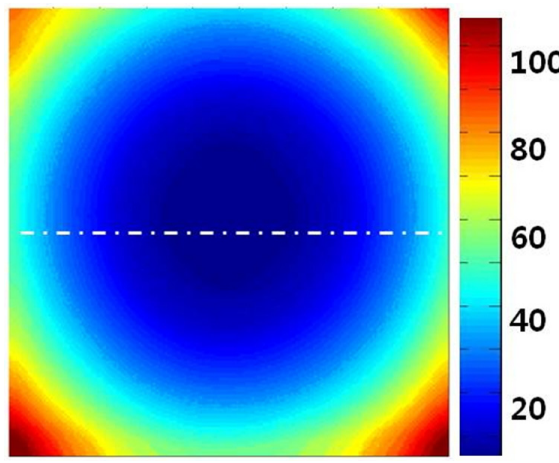

(c)

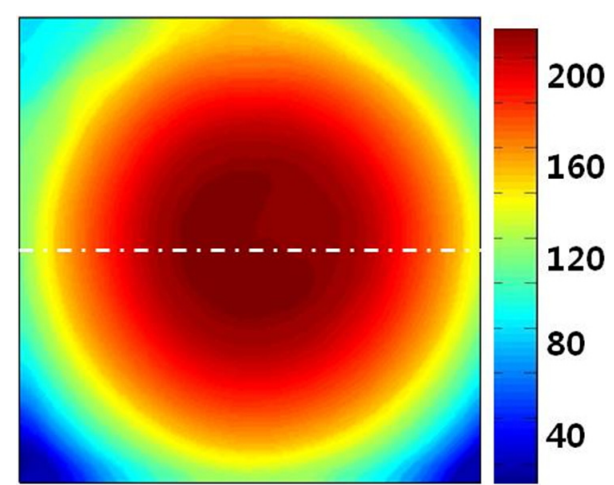

(b)

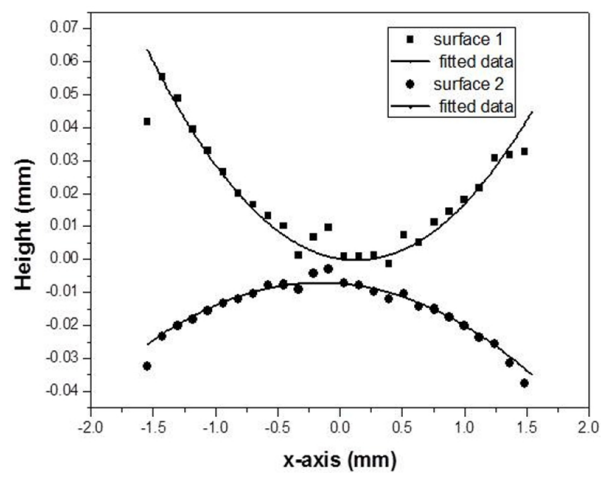

(d)

FIG. 5. Surface profiles of the achromatic lens. (a) Optical-path difference (height) for 532 and $633 \mathrm{~nm}$. (b) and (c) 3D color images of the front and back surface profiles. (d) Calculated and fitted profiles of each surface for the dashed lines in (b) and (c). 
where $a$ and $b$ are the coefficients of the curve. The ROC is given by $\mathrm{ROC}=\sqrt{(1 / a)+\left(b^{2} / 4 a^{2}\right)}$. The calculated ROC values for the surfaces were $28.47 \pm 1.22$ and $60.54 \pm 1.46$ $\mathrm{mm}$. These values were reasonably close to the design values, indicating that the proposed dual-wavelength DHM with liquids can be used for simultaneous measurement of the front and back profiles of a sample. Thus, unlike the many techniques available for measuring the surface profiles separately, the method described in this research provides the means to measure these profiles simultaneously. In this study we assume that the refractive indices of the studied materials are known; in future work we will investigate the case in which the refractive index of the sample is not known, or has a distribution.

\section{CONCLUSION}

Digital holographic microscopy can be used to measure optical-path differences with a high degree of accuracy. The optical-path difference depends on the refractive index and morphology of the samples. Normally optical components have a two-sided morphology, with front and back surfaces. We cannot determine the individual surface profiles with normal transmission DHM, because the output wave from the object presents integrated information. This study proposed a new experimental method and models for determining the individual surface profiles simultaneously using transmission DHM and liquids. We integrated dual-wavelength DHM with liquids and demonstrated a new method for determining the individual surface profiles of an achromatic lens, which has different ROC surfaces. The proposed method involves recording holograms at different wavelengths and then numerically reconstructing the phase information of the front and back 3D information of the sample. The proposed method can be applied to normal transmission interferometry for determining the 3D surface profiles of transmitted optical components.

\section{REFERENCES}

1. E. Cuche, P. Marquet, and C. Depeursinge, "Simultaneous amplitude-contrast and quantitative phase-contrast microscopy by numerical reconstruction of Fresnel off-axis holograms," Appl. Opt. 38, 6994-7001 (1999).

2. L. Xu, X. Peng, J. Miao, and A. Asundi, "Studies of digital microscopic holography with applications to microstructure testing," Appl. Opt. 40, 5046-5051 (2001).

3. M.-O. Jeong, N. Kim, and J.-H. Park, "Elemental image synthesis for integral imaging using phase-shifting digital holography," J. Opt. Soc. Korea 12, 275-280 (2008).

4. M. A. Kronrod, N. S. Merzlyakov, and L. P. Yaroslavski, "Reconstruction of a hologram with a computer," Sov. Phys. Tech. 17, 434-444 (1972).
5. L. P. Yaroslavskii and N. S. Merzlyakov, Methods of Digital Holography (Consultants Bureau, New York, USA, 1980).

6. L. Onural and P. D. Scott, "Digital decoding of in-line holograms," Opt. Eng. 26, 261124 (1987).

7. J. W. Goodman, Introduction to Fourier Optics, 2nd ed., Goodman ed. (McGraw Hill, New York, USA, 2005), Chapter 9.

8. U. Schnars and W. Juepther, Digital Holography, U. Schnars and W. Juepther eds. (Springer, Heidelberg, 2005).

9. L. Xu, J. Miao, and A. Asundi, "Properties of digital holography based on in-line configuration," Opt. Eng. 39, 32143219 (1999).

10. C. Depeursinge, Digital Holography and Three-dimensional Display, T. C. Poon ed. (Springer, New York, USA, 2006).

11. M. Schmitt, "Optical coherence tomography," IEEE Select. Topics Quantum Electron. 5, 1205-1215 (1999).

12. R. Leitgeb, C. Hitzenberger, and A. Fercher, "Performance of fourier domain vs. time domain optical coherence tomography," Opt. Express 11, 889-894 (2003).

13. I. Yamaguchi, T. Ida, M. Yokota, and K. Yamashita, "Surface shape measurement by phase shifting digital holography with wavelength shift," Appl. Opt. 45, 7610-7616 (2006).

14. B. Rappaz, F. Charrière, C. Depeursinge, P. J. Magistretti, and P. Marquet, "Simultaneous cell morphometry and refractive index measurement with dual wavelength digital holographic microscopy," Opt. Lett. 33, 744-746 (2008).

15. Y. Park, T. Yamauchi, W. Choi, R. Dasari, and M. S. Feld, "Spectroscopic phase microscopy for quantifying hemoglobin concentration in intact red blood cells," Opt. Lett. 34, 36683670 (2009).

16. C. Polhemus, "Two-wavelength interferometry," Appl. Opt. 12, 2071-2074 (1973).

17. H. J. Cho, D. C. Kim, Y. H. Yu, W. K. Jung, and S. H. Shin, "Dual-wavelength digital holography microscopy for BGA measurement using partial coherence source," J. Opt. Soc. Korea 15, 352-356 (2011).

18. G. Popescu, Quantitative Phase Imaging of Cells and Tissues (McGraw-Hill Professional, China, 2011).

19. K. Lee, K. Kim, J. Jung, J. Heo, S. Cho, S. Lee, G. Chang, Y. Jo, H. Park, and Y. Park, "Quantitative phase imaging techniques for the study of cell pathophysiology: From principles to application," Sensors 13, 4170-4191 (2013).

20. P. Marquet, B. Rappaz, P. J. Magistretti, E. Cuche, Y. Emery, T. Colomb, and C. Depeursinge, "Digital holographic microscopy: A noninvasive contrast imaging technique allowing quantitative visualization of living cells with subwavelength axial accuracy," Opt. Lett. 30, 468-470 (2005).

21. B. Rappaz, P. Marquet, E. Cuche, Y. Emery, C. Depeursinge, and P. J. Magistretti, "Measurement of the integral refractive index and dynamic cell morphometry of living cells with digital holographic microscopy," Opt. Express 13, 9361-9373 (2005).

22. K. Seong, "Optical metrology for transmission interferometric testing," Ch 4, Ph. D. Dissertation, University of Arizona (2008).

23. D. C. Ghiglia and M. D. Pritt, Two-Dimensional Phase Unwrapping (John Wiley \& Sons, New York, USA, 1998), Chapter 4. 